\title{
A multimodal approach to bus frequency design
}

\author{
M. Gallo' ${ }^{1}$ L. D’Acierno ${ }^{2}$ \& B. Montella ${ }^{2}$ \\ ${ }^{I}$ Department of Engineering, University of Sannio (Benevento), Italy \\ ${ }^{2}$ Department of Transportation Engineering, \\ 'Federico II' University of Naples, Italy
}

\begin{abstract}
In this paper we propose a model for optimising bus frequencies under the assumption of elastic demand, considering explicitly the effects of changes in transit supply on modal split. Indeed, neglecting demand elasticity may lead to solutions that may not represent actual design objectives. Using an objective function that is a weighted sum of user costs on all transportation systems (car and bus), operation costs and external costs, we propose a heuristic solution algorithm that is able to solve the problem in acceptable computing times, also for real-scale problems. The model and the algorithm are tested on a large urban multimodal network.
\end{abstract}

Keywords: transit network design, bus frequencies, elastic demand, optimisation models.

\section{Introduction}

The Transit Network Design Problem (see also Ceder and Wilson [1]) consists in optimising the main features of a transit network (line routes, frequencies, timetables, etc.). This problem has been extensively studied in the literature, some recent reviews being available in Guihaire and Hao [2] and Desaulniers and Hickman [3].

In this field, a major sub-problem is to design transit frequencies under the assumption that line routes are known and only service frequencies have to be designed. Several authors have focused on optimising transit frequencies, amongst whom Salzborn [4, 5], Furth and Wilson [6], Han and Wilson [7] 
Constantin and Florian [8], Claessens et al. [9] Gao et al. [10], Goossens et al. [11, 12], Schoebel and Scholl [13], Borndorfer et al. [14], and Yu et al. [15]. All the above papers adopt the assumption of rigid demand. This hypothesis is acceptable in practical applications where an existing network has to be (re)designed without allowing for new investments in the transit system. In this case, improvements in network performance are usually unable to significantly influence user mode choices. However, the assumption of rigid demand is inappropriate if transit services can be significantly improved with new investments. In such cases, one of the main design aims is precisely to shift users from road systems to transit systems and therefore the simulation of demand elasticity is indispensable. Recent contributions on transit network design problems with elastic demand have been proposed by Lee and Vuchic [16], Cipriani et al. [17] Fan and Machemehl [18, 19], Marín and García-Ródenas [20], and Gallo et al. [21].

In this paper we study the problem of optimising the frequencies of an urban bus system under the assumption of elastic demand. The paper is structured as follows: Section 2 focuses on model formulation; the solution algorithm is proposed in Section 3; numerical results on a real-scale network are reported in Section 4; Section 5 draws the main conclusions.

\section{Optimisation model}

To correctly simulate the elastic demand and evaluate the optimal frequencies of a bus network, we have to consider three transportation systems:

- bus system;

- private car system;

- pedestrians.

We assume that the topological configuration of the bus network is known. Index $l$ indicates the generic bus line and the frequency on line $l$ is indicated by $\varphi_{l}$. Vector $\varphi$ collects all frequencies. The bus network is partitioned into bus links, where each link represents the part of the network between two bus stops. Index $s$ indicates the generic bus link, and the bus user flow on link $s$ of a line $l$ is indicated by $f b_{s, l}$. Vector $f_{b}$ * collects all bus user flows. The length of a transit link is represented by $L b_{s}$ and the average commercial speed of a link is represented by $S b_{s}$.

We assume that the road network is known. Each road link, $k$, is associated to a capacity, $C a p_{k}$, a length, $L c_{k}$, a free-flow speed, $S c^{0}{ }_{k}$, and the parameters of a BPR cost function, $\alpha_{k}$ and $\beta_{k}$. The flows on a link $k$ are indicated by $f c_{k}$ and vector $f_{c}$ * collects all car user flows.

Each road link is also associated to a pedestrian speed assumed equal to 4 $\mathrm{km} / \mathrm{h}$ for simulating the access/egress to/from bus stops. Other links that can be used only by pedestrians can be added to the network (pedestrian areas, stairs, etc.). 
The decision variables are the bus line frequencies, $\varphi$; they could be assumed as continuous variables but in our problem we prefer to consider them as discrete variables.

We assume that the frequency of a line $l$ is between 1 and 15 buses $/ \mathrm{h}$ and that it is discrete:

$$
\begin{array}{ll}
1 \leq \varphi_{l} \leq 15 & \forall l \\
\varphi_{l} \text { is discrete } & \forall l
\end{array}
$$

Under such assumptions, the problem can be formulated with the following constrained discrete optimisation model that can be expressed as:

$$
\boldsymbol{\varphi}^{\wedge}=\arg _{\boldsymbol{\varphi}} \min w\left(\boldsymbol{\varphi} ; \boldsymbol{f}_{b}{ }^{*}, \boldsymbol{f}_{c}{ }^{*}\right)
$$

subject to:

$$
\begin{gathered}
1 \leq \varphi_{l} \leq 15 \quad \forall l \\
\varphi_{l} \text { is discrete } \quad \forall l \\
\sum_{l} \text { Ceiling }\left(\varphi_{l} r t_{l}\right) \leq N B_{\max } \\
\sum_{l} \varphi_{l} L_{l} \leq B K_{\max } \\
\max _{s} f b_{s, l} \leq \varphi_{l} B \text { Cap }{ }_{l} \forall l \\
{\left[\boldsymbol{f}_{b}{ }^{*}, \boldsymbol{f}_{c}{ }^{*}\right]=\Psi\left(\varphi, \boldsymbol{f}_{b}^{*}, \boldsymbol{f}_{c}^{*}\right)}
\end{gathered}
$$

where: $r t_{l}$ is the route time of line $l ; N B_{\max }$ is the maximum available number of buses; function Ceiling $(x)$ gives the smallest integer $\geq x ; L_{l}$ is the length of line $l$; $B K_{\max }$ is the maximum number of bus-kms that can be operated; $B C a p_{l}$ is the capacity of buses on bus line $l ; \boldsymbol{\Psi}($.) represents the multimodal assignment function.

Eqns (2) and (3) represent frequency constraints, eqns (4) and (5) represent budget constraints (on maximum number of buses available and maximum bus-kms operable respectively), eqn (6) is the capacity constraint on the maximum number of users per bus link and eqn (7) represents the multimodal assignment constraint able to jointly estimate equilibrium user flows on all transportation systems (see D'Acierno et al. [22]).

The objective function, $w($.$) , is a weighted sum of operator costs, O C($.$) ,$ transit user costs (bus and pedestrian), TUC(.), car user costs, $C U C($.$) and$ external costs, $E C($.).

Operator costs can be calculated as:

$$
O C(\varphi)=\left(\sum_{l} \varphi_{l} L_{l}\right) \cdot c_{b k m}
$$

where $c_{b k m}$ is the cost per bus-km ( $€ /$ bus-km) that we assume constant for all bus lines. In this model we do not include operator fixed costs since we may consider them independent of bus frequencies, hence irrelevant in the optimisation process. Indeed, we consider existing bus operators and we take into account the maximum number of buses available with the constraint (4) (we assume that no new buses can be acquired).

Transit user costs can be calculated as follows: 


$$
\begin{gathered}
\operatorname{TUC}\left(\boldsymbol{\varphi} ; \boldsymbol{f}_{b} *\right)=\beta_{b b}\left(\sum_{s, l} f b_{s, l} *(\boldsymbol{\varphi}) \cdot L b_{s} / S b_{s}\right)+\beta_{b w}\left(\sum_{g g}, f b w_{g g}, *(\boldsymbol{\varphi}) \cdot w b_{g g}(\boldsymbol{\varphi})\right)+ \\
+\beta_{a e}\left(\sum_{o g} f b a_{o g} *(\boldsymbol{\varphi}) \cdot a b_{o g}+\sum_{g^{\prime} d} f b e_{g^{\prime}{ }^{*}} *(\boldsymbol{\varphi}) \cdot e b_{g^{\prime} d}\right)+ \\
+\beta_{t r}\left(\sum_{n} f t_{n} * w t_{n} \cdot\left(n_{t r}-1\right)\right)
\end{gathered}
$$

where

$\beta_{b b} \quad$ is the perceived value of time spent on board a bus service $(€ / \mathrm{h})$;

$\beta_{b w} \quad$ is the perceived value of time spent waiting for a bus service $(€ / \mathrm{h})$;

$\beta_{a e} \quad$ is the perceived value of time of access to/egress from a bus stop $(€ / \mathrm{h})$;

$\beta_{t r} \quad$ is the perceived value of time spent transferring between bus services $(€ / \mathrm{h})$;

$f b_{s, l} * \quad$ represents the number of users (pass/h) on bus link $s$ of bus line $l$;

$L b_{s} \quad$ is the length $(\mathrm{km})$ of bus link $s$;

$S b_{s} \quad$ is the average commercial speed $(\mathrm{km} / \mathrm{h})$ on bus link $s$;

$f b w_{g g}, * \quad$ represents the number of users boarding at bus stop $g$ and alighting at bus stop $g^{\prime}$ (pass/h);

$w b_{g g}$, represents the waiting time for users boarding a bus line at bus stop $g$ and alighting from the same line at bus stop $g^{\prime}(h)$;

$f b a_{o g} * \quad$ represents the number of users accessing bus stop $g$ from origin $o$ ( $\mathrm{pass} / \mathrm{h})$;

$a b_{o g} \quad$ is the average access time between origin $o$ and bus stop $g(\mathrm{~h})$;

$f b e_{g}{ }^{\prime} *$ represents the number of users that egress from bus stop $g$ ' to destination $d$ (pass/h);

$e b_{g^{\prime} d} \quad$ is the average egress time between bus stop $g$ ' and destination $d(\mathrm{~h})$;

$f t_{n}{ }^{*} \quad$ represents the number of users that travel using $n$ different bus lines;

$w t_{n} \quad$ is the virtual time spent transferring between two bus lines;

$n_{t r} \quad$ is the number of bus lines used for travelling from the origin to the destination.

In particular, we assume that transit costs are not dependent on passenger flows, as usually assumed for transit systems. The costs of all parts of the trip (access, waiting, on-board, egress) are estimated in terms of time and converted into monetary costs by parameters $\beta$, representing the values of time (VOT). These parameters generally differ for the various parts of the trip since they are perceived by users with different weights: normally, waiting and access/egress times assume higher VOT values than that of on-board time. Waiting times, $w b_{g g}$, are estimated according to the hyperpath approach (Nguyen et al. [23]), taking into account the cumulative frequencies of attractive lines connecting an OD pair. Moreover, to allow for the discomfort associated to changing bus lines we consider a virtual time term spent on any transfer operation where the number of transfers can be easily calculated as the number of bus lines used minus 1 .

Car user costs can be calculated as:

$$
C U C\left(\varphi ; f_{c}^{*}\right)=\sum_{k}\left(f c_{k} *(\varphi) \cdot \delta_{c o} \cdot \beta_{t k} \cdot t c_{k}\left(f_{c_{k}} *(\varphi)\right)+f c_{k} *(\varphi) \cdot m c_{k} \cdot L_{k}\right)
$$

where

$f c_{k} *$ represents the car flows on road link $k(\mathrm{veh} / \mathrm{h})$; 
$\delta_{c o}$ is the average car occupancy factor (pass/veh);

$\beta_{t k}$ is the value of time for car users on road link $k(€ / \mathrm{h})$;

$t c_{k}$ is the travel time on road link $k(\mathrm{~h})$;

$m c_{k}$ is the monetary cost per km for running link $k(€ / \mathrm{km})$;

$L_{k} \quad$ is the length of road link $k(\mathrm{~km})$.

Car user costs is a weighted sum of travel time and monetary costs; the travel time is borne by all occupants of the car and is thus the product of car flow by occupancy factor by travel time. By contrast, the monetary costs are paid by each vehicle and hence amount to the product of cost per km by link length by car flow.

The external costs can be calculated as:

$$
E C\left(\boldsymbol{\varphi} ; \boldsymbol{f}_{c}^{*}\right)=e c_{k m} \cdot \sum_{k} f_{c_{k}} *(\boldsymbol{\varphi}) \cdot L_{k}
$$

where $e c_{k m}$ is the average value of external costs produced by a car per $\mathrm{km}$ travelled $(€ / \mathrm{km})$.

Hence the objective function is given by:

$$
w\left(\boldsymbol{\varphi} ; \boldsymbol{f}_{b}^{*}, \boldsymbol{f}_{c}^{*}\right)=O C(\boldsymbol{\varphi})+T U C\left(\boldsymbol{\varphi} ; \boldsymbol{f}_{b}^{*}\right)+C U C\left(\boldsymbol{\varphi} ; \boldsymbol{f}_{c}^{*}\right)+E C\left(\boldsymbol{\varphi} ; \boldsymbol{f}_{c}^{*}\right)
$$

\section{Solution algorithm}

The proposed algorithm is heuristic, consisting of five phases described in the following.

\section{Phase 1 - Unconstrained Mono-Dimensional Optimisation (UMDO)}

In this phase the frequency of each line $l$ is optimised, assuming that the values of other frequencies are constant, for instance equal to the current values or equal to a specific average value (e.g. 3 buses/h). Even if only 15 values can be assumed by each variable and an exhaustive search were possible in feasible computing times, as shown in a previous paper (Gallo et al. [21]), to reduce calculation times we may adopt a different approach which consists in analysing only nearest solutions (at most 2): if they improve the objective function value the neighbourhood search is reiterate; otherwise the local optimum is reached and the mono-dimensional optimisation for that frequency is completed.

However, in this phase we neglect budget constraints and capacity constraints, since the other line frequencies are not definitive and some active constraints should be inactive in the final configuration. We will take account of all constraints in the next phases.

Phase 2 - Unconstrained Starting Solution definition (USS)

The definition of the first starting solution is obtained by setting each frequency at the optimal value calculated in the first phase. From this solution the next phase will start without verifying whether that solution satisfies all constraints (obviously the assignment constraint always has to be satisfied).

Phase 3 - Unconstrained Neighbourhood Search Optimisation (UNSO) 
This phase searches for the local optimum nearest to the starting solution with a Neighbourhood Search (NS) algorithm, neglecting the constraints except that of assignment. Having set $\varphi^{i t}$ the solution at iteration it, its neighbourhood is the set of solutions, $N\left(\varphi^{i t}\right)$, that can be generated from the current solution by a single move, obtained by changing the frequency of a single line. The size of the neighbourhood should be as small as possible, since any time we generate and evaluate a solution a multimodal assignment has to be performed. Therefore we propose to generate the neighbourhood of a solution by considering as feasible moves for each line only those that modify the frequencies to the nearest (lower and upper) values. A neighbourhood can thus contain at most $2 \times n_{l}$ solutions (the number could be lower if some frequencies lie on the boundary of feasible solutions).

A neighbourhood search algorithm can adopt two different approaches for generating the next solution:

- the Steepest Descent (SD) method, which examines and evaluates all neighbouring solutions, $N\left(\varphi^{i t}\right)$, and chooses the best solution as the next one;

- the Random Descent (RD) method which randomly extracts a solution from the neighbourhood, $N\left(\varphi^{i t}\right)$, and evaluates only its objective function; if the new solution is better than the current one, it becomes the next solution; otherwise, another neighbourhood solution is randomly extracted and so on. If no neighbourhood solutions improve the objective function, the search ends and the last solution is a local optimum.

In these initial phases we do not consider constraints (except that of assignment) because the aim is to search for distant solutions; all constraints will be considered in subsequent phases.

\section{Phase 4 - Constrained Starting Solution definition (CSS)}

In this phase we analyse all solutions generated in the previous phases, selecting the one that minimises the objective function and jointly satisfies all constraints. At least the current solution (the first frequency values adopted in phase 1) is feasible for real cases.

\section{Phase 5 - Constrained Neighbourhood Search Optimisation (CNSO)}

Finally, in this phase we perform a neighbourhood search taking into account only solutions in each neighbourhood that satisfy all constraints. In this way we steer the search only towards feasible solutions. Also in this case we can adopt the SD or RD approach.

Although we could adopt both SD and RD methods for phases 3 and 5, in a previous paper it was shown that the RD approach performed successfully for solving the Road Network Design Problem (Gallo et al. [24]). Hence comparison between $\mathrm{SD}$ and $\mathrm{RD}$ approaches will be provided only with respect to constrained optimisation (i.e. phase 5).

\section{Numerical results}

The proposed model and algorithm were tested on a real-scale network representing the transportation network of Salerno, a city in the south of Italy 
with about 140,000 inhabitants. The main features of the network are reported in Figures 1 and 2, and in Table 1. In particular, the peak-hour (all-mode) demand is $35,909 \mathrm{users} / \mathrm{h}$.

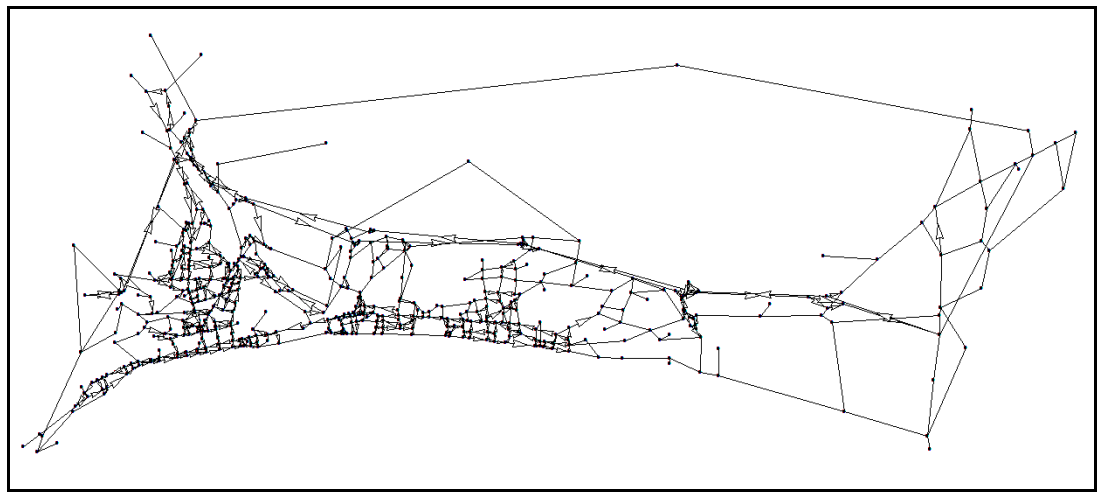

Figure 1: $\quad$ Salerno road network.

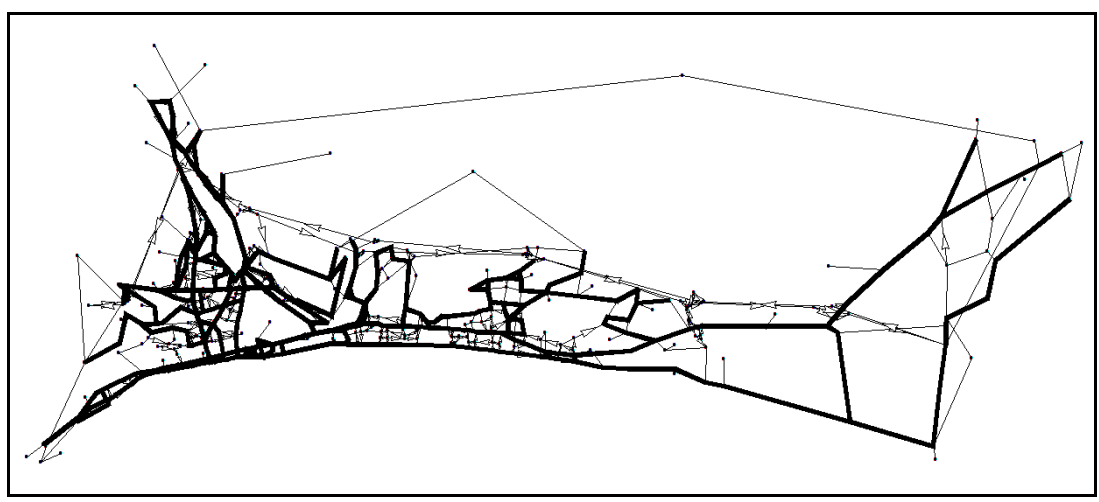

Figure 2: Salerno transit network.

The proposed algorithm was tested by considering the frequencies of all 40 bus lines as decision variables, and assuming a new investment in terms of transit operational costs of $+20 \%$ which corresponds to setting constraint (5) at $1,614.36$ bus- $\mathrm{km} / \mathrm{h}$ (indeed, the value of bus- $\mathrm{kms}$ corresponding to the initial solution was $1,345.30$ bus $-\mathrm{km} / \mathrm{h}$ ). The new value of constraint (5) in terms of operating costs corresponds to a maximum cost of public transport of $6,134.57$ $€ / \mathrm{h}$ while the cost corresponding to the initial frequencies is $5,112.40 € / \mathrm{h}$.

Table 1: $\quad$ Test network features.

\begin{tabular}{cccccc}
\hline & Links & Line links & Pedestrian links & Nodes & Transit lines \\
\hline Road network & 1,133 & - & - & 529 & - \\
\hline Transit network & 7,124 & 3,141 & 1,326 & 3,779 & 40 \\
\hline
\end{tabular}


In terms of the algorithm framework, as motivated in the last part of the previous section, we tested two versions of the proposed algorithm: the RD version and the SD version. The differences in the implemented approaches are related to the descent method adopted in phase 5 .

Figures 3 and 4 show algorithm performances, in terms of analysed solutions and related objective function values, in the case of the SD and RD approach, while Figure 5 provides a comparison between the proposed algorithm approaches.

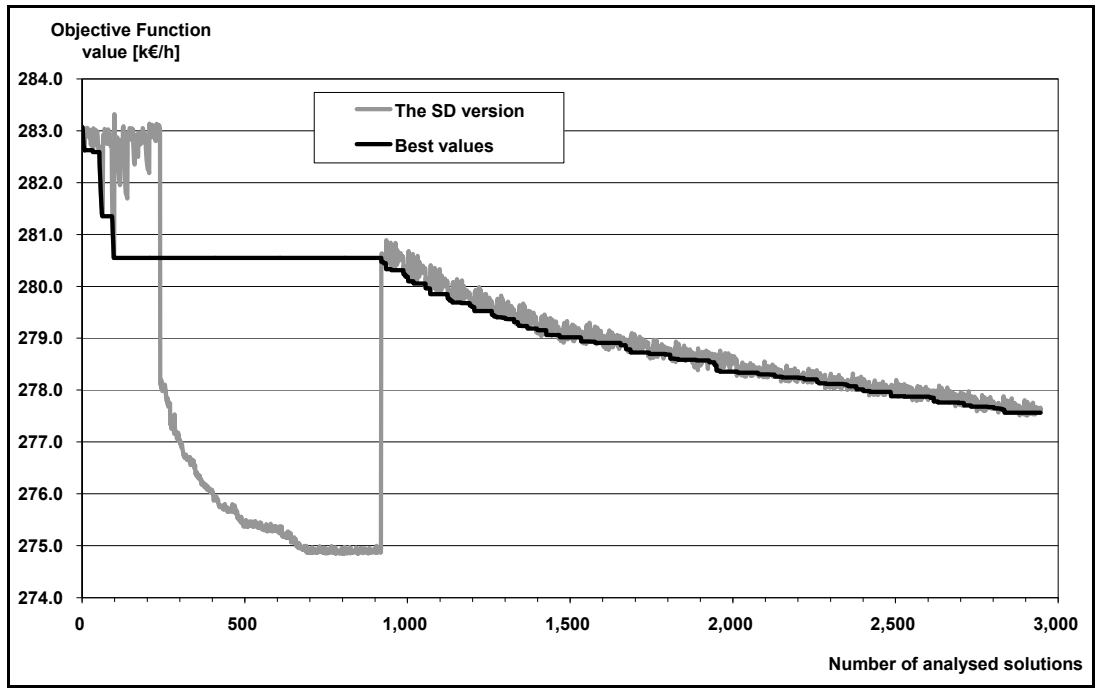

Figure 3: Algorithm performance in the case of the SD version.

It is worth noting that both algorithms converged to a local optimal solution: the SD version required 113 hours of computing times versus 42 hours of RD version. Particularly, SD version evaluated 2,946 solutions while RD version 1,106 solutions.

The local optimal solutions generated by the two versions of the algorithms differ greatly (see Table 2): the distance between solutions is 25 (i.e. 25 elementary moves are required to transform a solution from $\mathrm{SD}$ to $\mathrm{RD}$ or back); the distance between the SD solution and the starting solution is 36 while it is 33 for the RD approach. Moreover, the SD approach generates a local optimum that reduces the objective function by $1.93 \%$ while for the $\mathrm{RD}$ approach there is a reduction of $1.62 \%$.

These results highlight the advantages of the algorithm based on the RD approach over the SD-based algorithm since the computing times are significantly lower. As regards the final solution, it being a local optimum, the fact that we obtain a better value with the SD is not a general result since objective function is not strictly convex. Thus an algorithm which follows a random direction ( $\mathrm{RD}$ version) could provide better results by exploring a wide solution space. 


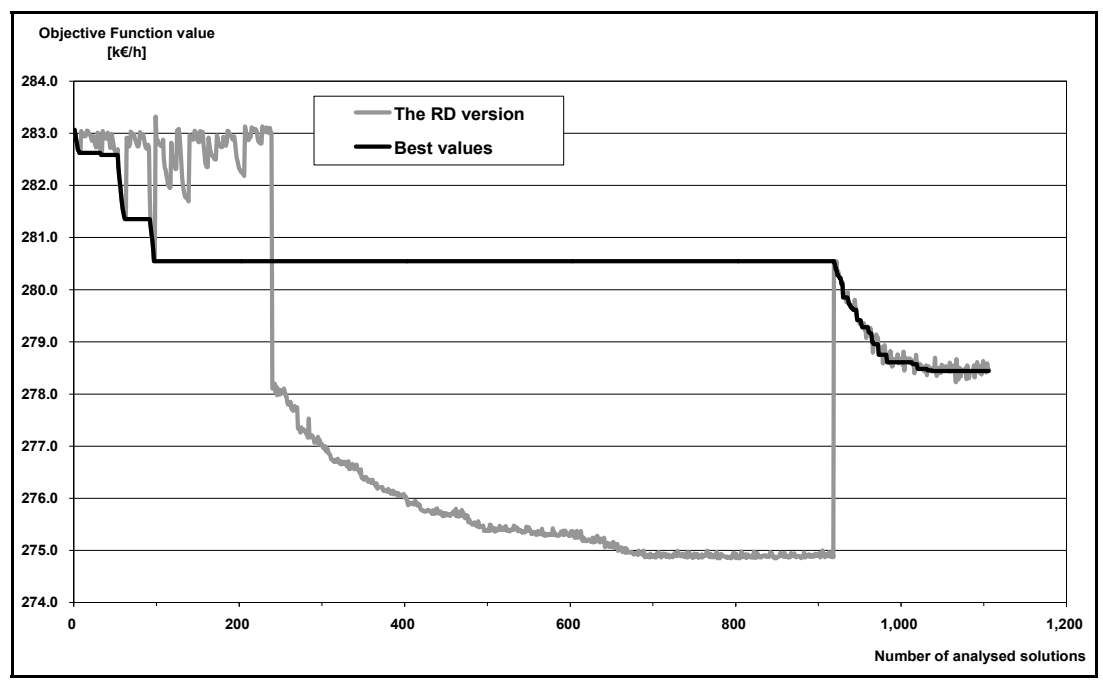

Figure 4: Algorithm performances in the case of RD version.

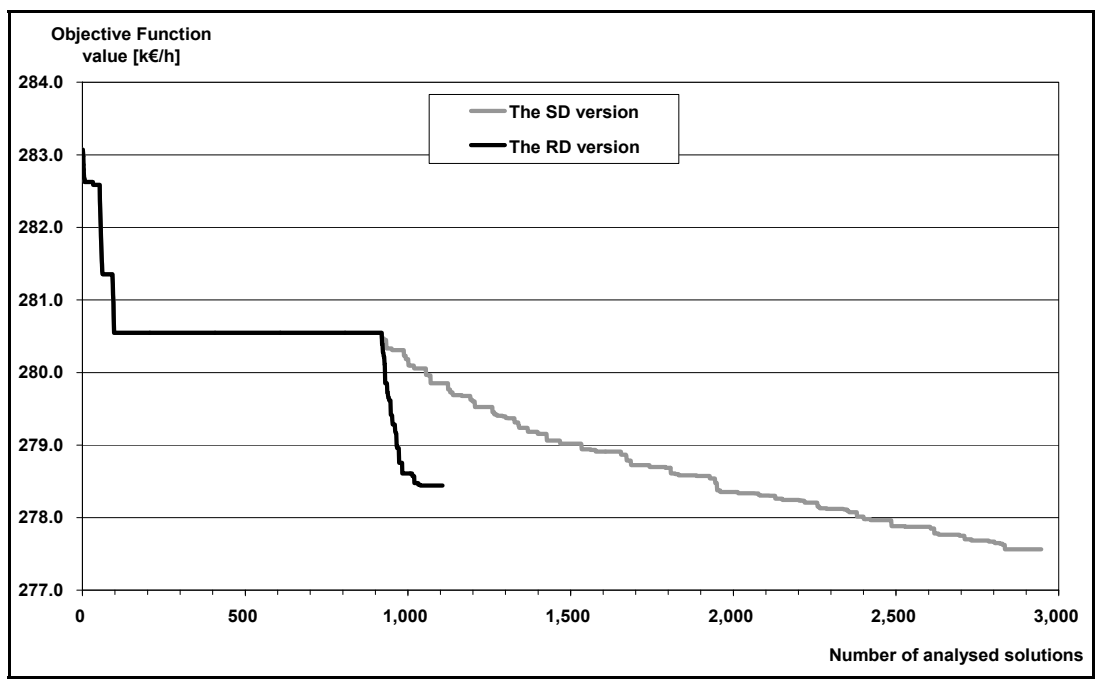

Figure 5: Comparison between algorithm approaches.

With regard to the model, an important result appears to be the analysis of effect of transit system investments (in our case in terms of bus-kms or equivalently in terms of operational cost subsidies). Indeed, as regards the solution found with the two proposed approaches, the increase in operator transit costs is slightly lower than the budget constraint (i.e. $+19.89 \%$ in the SD approach vs. $+19.77 \%$ in the RD case); ticket revenues increase by $2.20 \%$ in the $\mathrm{SD}$ case and $1.95 \%$ in $\mathrm{RD}$; transit user travel times decrease respectively by 
$1.98 \%$ and $1.68 \%$; monetary costs of road users decrease respectively by $2.42 \%$ and $2.14 \%$; travel times for the same users decrease by $4.48 \%$ and $3.86 \%$; external costs are reduced by $1.97 \%$ and $1.65 \%$, respectively, in cases SD and RD. Finally, in terms of transit system travel demand we obtain a respective increase of $2.21 \%$ and $1.95 \%$.

Table 2: $\quad$ Local optimal solutions.

\begin{tabular}{|c|c|c|c|c|c|c|c|c|c|c|}
\hline Line & 1 & 2 & 3 & 4 & 5 & 6 & 7 & 8 & 9 & 10 \\
\hline Starting solution & 3 & 2 & 4 & 1 & 1 & 5 & 2 & 4 & 4 & 3 \\
\hline $\begin{array}{l}\text { Final solution with SD } \\
\text { approach }\end{array}$ & 3 & 1 & 4 & 1 & 1 & 5 & 2 & 4 & 8 & 4 \\
\hline $\begin{array}{c}\text { Final solution with RD } \\
\text { approach }\end{array}$ & 4 & 1 & 5 & 1 & 1 & 5 & 3 & 4 & 5 & 3 \\
\hline Line & 11 & 12 & 13 & 14 & 15 & 16 & 17 & 18 & 19 & 20 \\
\hline Starting solution & 4 & 4 & 3 & 3 & 1 & 1 & 1 & 1 & 2 & 1 \\
\hline $\begin{array}{l}\text { Final solution with SD } \\
\text { approach }\end{array}$ & 4 & 5 & 3 & 4 & 8 & 1 & 1 & 1 & 5 & 1 \\
\hline $\begin{array}{c}\text { Final solution with RD } \\
\text { approach }\end{array}$ & 4 & 5 & 3 & 3 & 8 & 1 & 2 & 1 & 1 & 1 \\
\hline Line & 21 & 22 & 23 & 24 & 25 & 26 & 27 & 28 & 29 & 30 \\
\hline Starting solution & 1 & 3 & 1 & 1 & 3 & 1 & 3 & 2 & 1 & 1 \\
\hline $\begin{array}{l}\text { Final solution with SD } \\
\text { approach }\end{array}$ & 1 & 3 & 1 & 1 & 3 & 2 & 6 & 2 & 1 & 1 \\
\hline $\begin{array}{l}\text { Final solution with RD } \\
\text { approach }\end{array}$ & 1 & 5 & 1 & 1 & 3 & 3 & 4 & 3 & 2 & 1 \\
\hline Line & 31 & 32 & 33 & 34 & 35 & 36 & 37 & 38 & 39 & 40 \\
\hline Starting solution & 2 & 8 & 2 & 3 & 5 & 6 & 6 & 4 & 4 & 3 \\
\hline $\begin{array}{l}\text { Final solution with SD } \\
\text { approach }\end{array}$ & 7 & 6 & 1 & 2 & 5 & 6 & 6 & 4 & 1 & 1 \\
\hline $\begin{array}{c}\text { Final solution with RD } \\
\text { approach }\end{array}$ & 5 & 6 & 2 & 3 & 5 & 6 & 5 & 4 & 1 & 1 \\
\hline
\end{tabular}

It is worth noting that the proposed multimodal approach for analysing transport policies (in our case the design of bus frequencies and the increase in public transport subsidies) together with all transportation systems also highlights indirect effects such as a decrease in road user travel times due to a reduction in road congestion (i.e. an increase in public transport travel demand reduces road system travel demand and hence congestion).

In terms of a contribution to the objective function value in the final solution we obtain that operational costs represent $2.21 \%$ and $2.20 \%$, respectively, in cases SD and RD; travel times of transit users contribute $46.96 \%$ in both cases; road system monetary costs, respectively, $40.48 \%$ and $40.47 \%$; road user travel times $6.49 \%$ and $6.51 \%$; finally, external costs account for $3.85 \%$ in both cases.

\section{Conclusions and research prospects}

This paper proposed a model and a heuristic algorithm to optimise bus frequencies under the assumption of elastic demand. The proposed model 
considers demand as elastic at mode choice level and an objective function that jointly evaluates the costs of car and transit users, operation costs and external costs. The model and algorithm were tested on a real scale problem. Our results show the applicability of the model also on real networks in terms of acceptable computing times for strategic planning.

Further research will aim to extend the model to multi-period simulation, to consider the line routes as decision variables, and to compare the proposed algorithm with other heuristic and meta-heuristic algorithms such as natureinspired algorithms.

\section{References}

[1] Ceder, A. \& Wilson, N.H.M., Bus network design. Transportation Research B, 20, pp. 331-344, 1986.

[2] Guihaire, V. \& Hao, J.-K., Transit network design and scheduling: A global review. Transportation Research A, 42, pp. 1251-1273, 2008.

[3] Desaulniers, G. \& Hickman, M., Public transit. Handbooks in Operation Research and Management Science: Transportation, 14, pp. 69-120, 2007.

[4] Salzborn, F.J.M., Optimum bus scheduling. Transportation Science, 6, pp. 137-148, 1972.

[5] Salzborn, F.J.M., Scheduling bus systems with interchanges. Transportation Science, 14, pp. 211-220, 1980.

[6] Furth, P.G. \& Wilson, N.H.M., Setting frequencies on bus routes: theory and practice. Transportation Research Record, 818, pp. 1-7, 1982.

[7] Han, A.F. \& Wilson, N., The allocation of buses in heavily utilized networks with overlapping routes. Transportation Research B, 16, pp. 221232, 1982.

[8] Constantin, I. \& Florian, M., Optimizing frequencies in a transit network: a nonlinear bi-level programming approach. International Transactions in Operational Research, 2, pp. 149-164, 1995.

[9] Claessens, M.T., van Dijk, N.M. \& Zwaneveld, P.J., Cost optimal allocation of rail passenger lines. European Journal of Operational Research, 110, pp. 474-489, 1998.

[10] Gao, Z., Sun, H. \& Shan, L., A continuous equilibrium network design model and algorithm for transit systems. Transportation Research $B, \mathbf{3 8}$, pp. 235-250, 2004.

[11] Goossens, J.-W., van Hoesel, S. \& Kroon, L., A Branch-and-Cut approach for solving railway line-planning problems. Transportation Science, 38, pp. 379-393, 2004.

[12] Goossens, J.-W., van Hoesel, S. \& Kroon, L., On solving multi-type railway line planning problems. European Journal of Operational Research, 38, pp. 403-424, 2006.

[13] Schoebel, A. \& Scholl, S., Line planning with minimal traveling time. Proceedings of 5th Workshop on Algorithmic Methods and Models for Optimization of Railways, Palma de Mallorca, Spain, 2005. 
[14] Borndorfer, R., Grotschel, M., Pfetsch, M.E., A column-generation approach to line planning in public transport. Transportation Science, $\mathbf{4 1}$, pp. 123-132, 2007.

[15] Yu, B., Yang, Z. \& Yao, J., Genetic algorithm for bus frequency optimization. Journal of Transportation Engineering, 136, pp. 576-583, 2010.

[16] Lee, Y.J. \& Vuchic, V.R., Transit network design with variable demand. Journal of Transportation Engineering, 131, pp. 1-10, 2005.

[17] Cipriani, E., Petrelli, M. \& Fusco, G., A multimodal transit network design procedure for urban areas. Advances in Transportation Studies, A10, pp. 5-20, 2006.

[18] Fan, W. \& Machemehl, R.B., Optimal transit route network design problem with variable transit demand: genetic algorithm approach. Journal of Transportation Engineering, 132, pp. 40-51, 2006.

[19] Fan, W. \& Machemehl, R.B., Tabu search strategies for the public transportation network optimisations with variable transit demand. Computer-Aided Civil and Infrastructure Engineering, 23, pp. 502-520. 2008.

[20] Marín, A. \& García-Ródenas, R., Location of infrastructure in urban railway networks. Computers \& Operations Research, 36, pp. 1461-1477, 2009.

[21] Gallo, M., Montella, B. \& D’Acierno, L., The transit network design problem with elastic demand and internalisation of external costs: an application to rail frequency optimisation. Transportation Research $C, \mathbf{1 9}$, 2011.

[22] D’Acierno, L., Montella, B. \& Gallo, M., Multimodal assignment to congested networks: fixed-point models and algorithms. Proceedings of 'European Transport Conference 2002', Cambridge, UK. 2002.

[23] Nguyen, S., Pallottino, S. \& Gendreau, M., Implicit enumeration of hyperpaths in a logit model for transit network. Transportation Science, 32, pp. 54-64, 1998.

[24] Gallo, M., D’Acierno, L. \& Montella, B., A meta-heuristic approach for solving the Urban Network Design Problem. European Journal of Operational Research, 201, pp. 144-157, 2010. 